\title{
Role of architecture in the elastic response of semiflexible polymer and fiber networks
}

\author{
Claus Heussinger and Erwin Frey \\ Arnold Sommerfeld Center for Theoretical Physics and CeNS, Department of Physics, Ludwig-Maximilians-Universität München, \\ Theresienstrasse 37, D-80333 München, Germany \\ and Hahn-Meitner-Institut, Glienicker Strasse 100, D-14109 Berlin, Germany
}

(Received 8 May 2006; revised manuscript received 21 August 2006; published 17 January 2007)

\begin{abstract}
We study the elasticity of cross-linked networks of thermally fluctuating stiff polymers. As compared to their purely mechanical counterparts, it is shown that these thermal networks have a qualitatively different elastic response. By accounting for the entropic origin of the single-polymer elasticity, the networks acquire a strong susceptibility to polydispersity and structural randomness that is completely absent in athermal models. In extensive numerical studies we systematically vary the architecture of the networks and identify a wealth of phenomena that clearly show the strong dependence of the emergent macroscopic moduli on the underlying mesoscopic network structure. In particular, we highlight the importance of the polymer length, which to a large extent controls the elastic response of the network, surprisingly, even in parameter regions where it does not enter the macroscopic moduli explicitly. Understanding these subtle effects is only possible by going beyond the conventional approach that considers the response of typical polymer segments only. Instead, we propose to describe the elasticity in terms of a typical polymer filament and the spatial distribution of crosslinks along its backbone. We provide theoretical scaling arguments to relate the observed macroscopic elasticity to the physical mechanisms on the microscopic and mesoscopic scales.
\end{abstract}

DOI: 10.1103/PhysRevE.75.011917

PACS number(s): 87.16.Ka, 62.20.Dc, 82.35.Pq

\section{INTRODUCTION}

Classical elasticity is a continuum theory that deals with the large-scale deformation properties of solid systems. It relates stresses and strains by introducing a host of phenomenological parameters-e.g., shear and bulk modulus for isotropic media - that characterize the elastic properties on wavelengths large compared with any other material length scale [1]. Biological systems like the cell or subcellular organelles are often characterized by a highly heterogeneous structure with a multitude of hierarchical levels of organization [2]. Due to these large-scale inhomogeneities, which may extend up to the scale of the system size, the applicability of elasticity theory on smaller length scales has to be critically examined. In particular, the actual deformations in the system are expected to relate to the externally applied stresses in a nontrivial way that crucially depends on the specific structural details.

To shed some light on the relevance of structure to the effective elasticity this article deals with the calculation of elastic constants in networks of semiflexible polymers. In eukaryotic cells these networks assemble to form the cytoskeleton, which plays a central role in many cellular functions such as locomotion, adhesion, or cell division. From the point of view of structure already a one-component isotropic solution of semiflexible polymers represents an interesting model system being studied for many years [3-5]. One of the main quantities of interest is the plateau value of the shear modulus found at intermediate time scales where singlepolymer bending fluctuations are equilibrated, yet center-ofmass motion is negligible. The generally accepted theory for the concentration dependence of the plateau modulus is based on the free energy change of confining a polymer to a tube $[3,6-8]$, the diameter of which is a consequence of the structural organization of the tubes in the form of a random assembly of cylinders [9]. Even though this has been well known for more than a decade, computer simulations to study the geometrical as well as elastic properties in this fibrous architecture have only recently been realized $[10,11]$.

Upon the addition of cross-linking agents or other regulating proteins one can induce structural changes to modify the network architecture in many ways [12-16]. There have been attempts to describe the phase diagram of these systems $[17,18]$; the detailed mechanisms that lead to a particular structure, however, are far from being understood. In general, there will be a complex interplay of polymer kinetics, thermal fluctuations, and chemical as well as mechanical properties of the polymers and the cross-linking agents yielding a particular architecture relevant for a given physical situation.

A complementary approach to describe cross-linked networks is to neglect these intricate "dynamic" aspects of the network and to concentrate on a "static" architecture and its effect on the macroscopic elasticity [19-24]. In the structural engineering community, for example, it is of tantamount importance to analyze the architecture of structures made of beams or trusses. A common way to take advantage of the reduced weight compared to the bulk material without suffering from a loss of stiffness is a triangulation of the basic cells. This eliminates the soft bending modes of the beams and makes it possible to construct huge cantilever bridges like that over the Firth of Forth in Scotland or towers like Eiffel's tower in Paris. Since the rigidity of these structures is not due to the individual beam but to a nonlocal backcoupling effect induced by the architecture of the network, the triangulation is therefore one example on how cooperativity among the building blocks may be possible.

To address this question of cooperativity in the context of the elasticity of cross-linked stiff polymer networks we will concentrate in the following on two generic structures, cellular and fibrous networks, which may serve as reference 

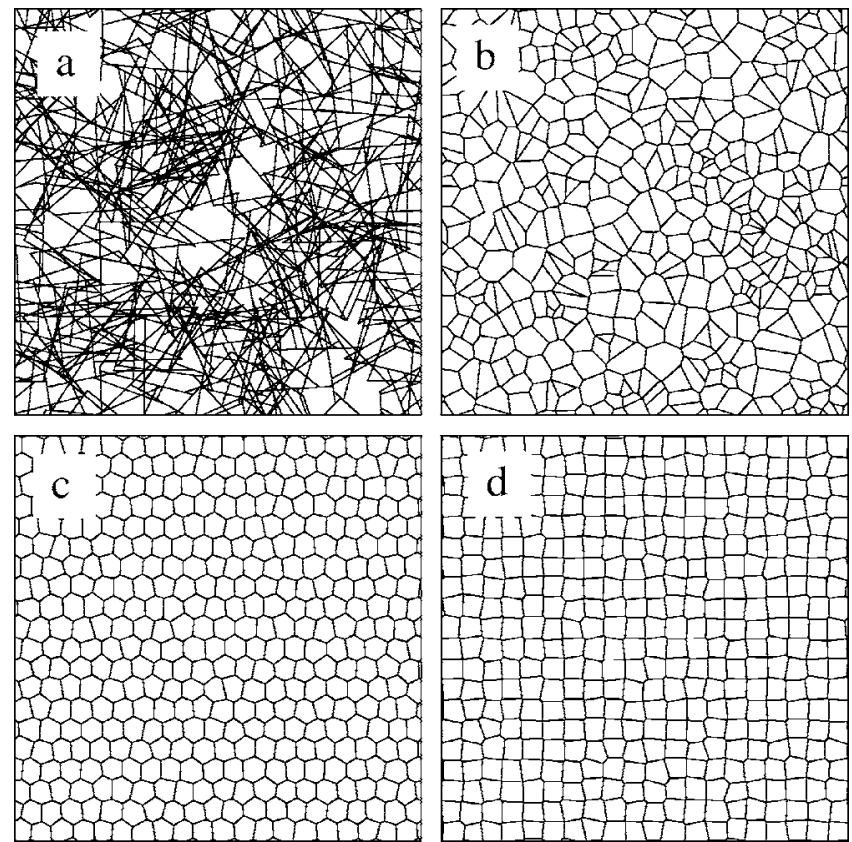

FIG. 1. Illustration of the different architectures of (a) fibrous and (b)-(d) cellular materials in two dimensions. While (a) and (b) are random structures generated by Poisson point processes, (c) and (d) are quite regular networks based on honeycomb and square lattices, respectively.

systems for the classification of real polymer networks. While cellular structures may be characterized by the amount of randomness in size and type of their unit cells [see Figs. 1(b)-1(d)], fibrous networks have a hierarchical structure, where smaller cells are generated within lager cells within even larger cells [Fig. 1(a)]. This is a consequence of the presence of the additional mesoscopic scale of the fiber length. As we will see, this length scale is ultimately responsible for the intricate scaling properties of the elasticity of fibrous polymer networks. The goal of this article is to identify these mechanisms, which couple the particular network structure to the properties of the individual polymers and effectuate the macroscopic elasticity of the system.

In contrast to the purely mechanical systems relevant for engineering applications [19-21,25], the systems we would like to study are immersed in a thermal environment. This implies that in addition to the usual enthalpic polymer elasticity also entropic effects have to be accounted for. We have published a brief account of this study recently [23]. It will turn out that by accounting for the entropic origin of the single-polymer elasticity, the networks acquire a strong susceptibility to polydispersity and structural randomness that is completely absent in athermal models.

The article is organized as follows. In Sec. II we motivate our modeling approach of thermally fluctuating networks of stiff polymers. This will lead us to the definition of effective elastic properties of the "polymer segments" that constitute the elementary building blocks of the network. In Secs. III and IV these polymer segments are assembled into cellular and fibrous networks, respectively. The macroscopic elastic constants of these structures are calculated and related to the particular architectural features. Finally, in Sec. V we present

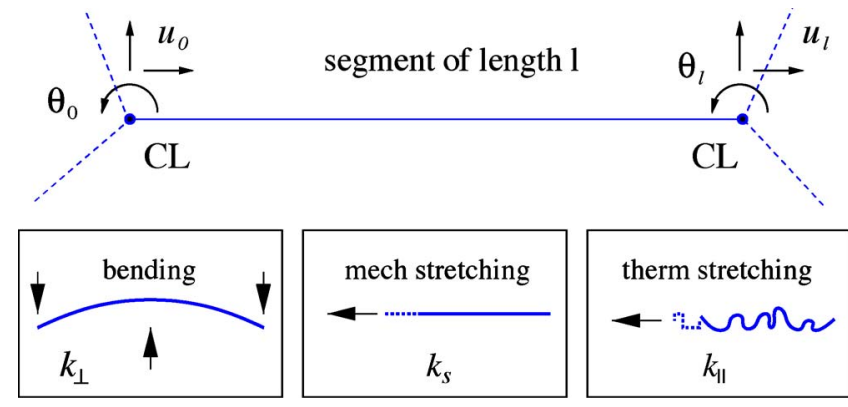

FIG. 2. (Color online) Illustration of a polymer segment of length $l$ and its connection to the network (dashed lines) at the cross-links (CL). The three degrees of freedom at each cross-link are denoted by $\mathbf{u}$ and $\theta$, respectively. Identification of the three possible modes of deformation and their stiffnesses $k_{\perp}, k_{s}$, and $k_{\|}$as defined in the text.

our main conclusions and hint at implications for experiments.

\section{MODEL DEFINITION}

To study the elastic properties of thermally fluctuating cross-linked stiff polymer networks we calculate numerically the low-frequency shear modulus. Assuming a time-scale separation between the fast bending fluctuations of the single polymer and their very slow center-of-mass motion, we adopt a description of the system in the spirit of a BornOppenheimer approximation. This neglects entropic contributions from the "slow variables," the cross-link positions, while assuming the "fast" polymer degrees of freedom to be equilibrated at all times. Macroscopic quantities will then depend parametrically on the set of cross-link variables. A macroscopic shear strain $\gamma$ constrains the cross-links at the boundaries, while those in the bulk are moving freely to minimize the elastic energy $E$. The shear modulus is defined as its second derivative with respect to the shear strain, $G$ $=V^{-1} \partial^{2} E_{\min } / \partial \gamma^{2}$, where $V$ is the system volume.

By keeping the positions of the cross-links fixed, the energy can be written as a sum,

$$
E=\sum_{\alpha} e\left(\boldsymbol{\delta} \mathbf{x}^{\alpha}\right),
$$

over contributions from individual polymer segments $\alpha$, each of which connects a given pair of cross-links (see Fig. 2). The single-segment energy $e$ depends on the generalized "displacement vector" $\boldsymbol{\delta} \boldsymbol{x}^{\alpha}$, which incorporates the degrees of freedom, displacements $\boldsymbol{u}$ and rotations $\boldsymbol{\theta}$, of the two cross-links pertaining to the segment.

In the numerical section we focus on two-dimensional systems such that a vector $\boldsymbol{\delta}_{2 \mathrm{D}}=\left(\boldsymbol{u}_{0}, \theta_{0}, \boldsymbol{u}_{l}, \theta_{l}\right)$ has six components. Those are in-plane displacements $\boldsymbol{u}_{0, l}$ and $z$-axis rotations $\theta_{0, l}$, at both ends $0, l$ of the segment with length $l$ (see Fig. 2). Note that the additional variable of cross-link rotation sets our system apart from bond-bending and related models [26] where only translational degrees of freedom are accounted for. As a consequence one also has to account for the presence of torques as the conjugate variable to rotations. 
To leading order, in linear elasticity, the single-segment quantity $e$ is a quadratic function of its coordinates,

$$
e(\mathbf{x})=\frac{1}{2} \mathbf{x}^{T} \mathbf{K} \mathbf{x},
$$

which defines the "stiffness matrix" $\mathbf{K}$ (spring constants) of the polymer strand.

In models of classical beams with cross-section radius $r$ the matrix elements are well established and relate to the two deformation modes of stretching (s) and bending $(\perp)$, respectively. While the former is characterized by the Young's modulus $E$ of the material, the latter depends on the bending stiffness $\kappa=E \pi r^{4} / 4$, here taken for circular cross sections. To calculate the bending response, standard Euler-Bernoulli beam theory [1] is used.

While we refer to the Appendix for a derivation of the complete matrix, it turns out that the response of a beam of length $l$ is sufficiently characterized already by two elements of $\mathbf{K}$,

$$
k_{s}(l)=4 \kappa / l r^{2}, \quad k_{\perp}(l)=3 \kappa / l^{3},
$$

relating to either deformation mode. Due to their small aspect ratios $r / l \ll 1$, slender rods are highly anisotropic and much softer in bending than in stretching, $k_{\perp} / k_{s} \propto(r / l)^{2}$. In this approximation the two deformation modes are decoupled such that, for example, prestretching does not influence the bending response. Therefore, Euler buckling cannot be accounted for.

Here, we consider thermally fluctuating stiff polymers immersed in a heat bath of solvent molecules. In these systems, the effects of temperature on the elastic properties of the polymer can be quantified by defining the persistence length $l_{p}$ as the ratio of bending stiffness to thermal energy $l_{p}$ $=\kappa / k_{B} T$. With this definition we have, in addition to the enthalpic stiffness of the classical beam, an entropic contribution

$$
k_{\|}(l)=\zeta \kappa \frac{l_{p}}{l^{4}}
$$

to the polymer's stretching compliance that can be calculated within the wormlike chain model $[28,27]$. The prefactor $\zeta$ depends on the specific boundary conditions chosen at the ends of the polymer segment. Its value can be absorbed in the persistence length and therefore only quantitatively affects the results. To avoid a large numerical offset with respect to Eq. (3), we have chosen $\zeta=6$, which corresponds to a boundary condition with one end clamped [27]. Having two longitudinal deformation modes $k_{s}$ and $k_{\|}$the effective stretching stiffness is equivalent to a serial connection

$$
k_{\mathrm{eff}}^{-1}=k_{s}^{-1}+k_{\|}^{-1} .
$$

Thus, the elastic properties of the polymer segments are given by the classical theory of beam bending supplemented by a generalized stretching stiffness, which also includes entropic effects. While the stiffness matrix has only been set up for the two-dimensional problem, the governing entries in three dimensions will still be the same Eqs. (3) and (4).

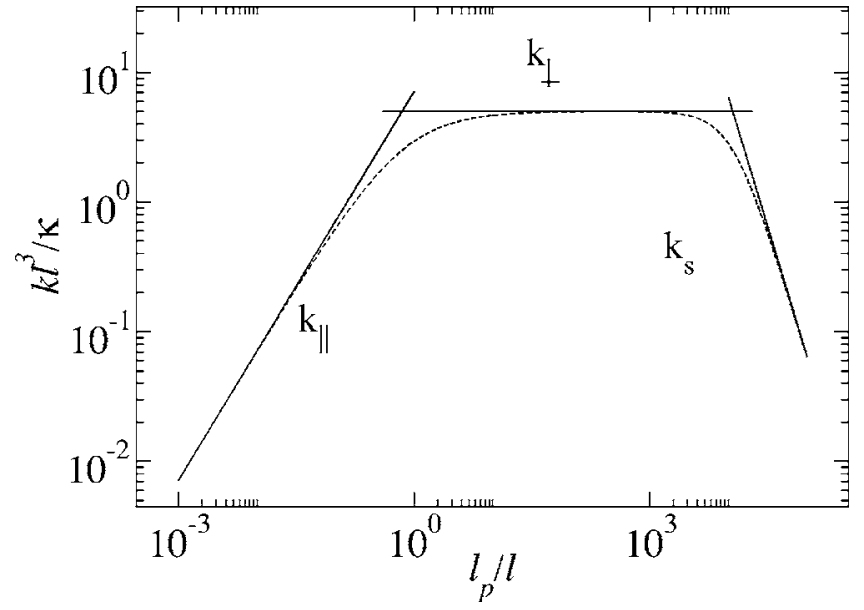

FIG. 3. Dependence of the three spring constants $k_{\|}, k_{\perp}$, and $k_{s}$ on persistence length $l_{p} / l$. The dashed line corresponds to a hypothetical spring with the three deformation modes connected in series.

As one can infer from Eqs. (3) and (4), at a given temperature $T$ there are two length scales characterizing the material properties of the polymers: the radius $r$ and the persistence length $l_{p}$. Typical biological polymers are characterized by a ratio $R=l_{p} / r \gg 1$. F-actin, for example, a key component of the cytoskeleton has $R=O\left(10^{4}\right)\left(r \approx 5 \mathrm{~nm}, l_{p} \approx 17 \mu \mathrm{m}\right)$, while microtubules, most important for cell division and intracellular transport, have an even larger $R=O\left(10^{6}\right)$. For specificity, we require in the following a constant $R=1.5$ $\times 10^{4}$; the precise value, however, is irrelevant if one is interested only in the thermal response where the radius does not enter and $k_{s} \rightarrow \infty .{ }^{1}$ Occasionally, we will perform this limit to highlight features that are independent of the mechanical stretching response. On the other hand, the location of the crossover point, where the mechanical stretching becomes relevant, does indeed depend on the choice of $R$. By definition, it determines the relative magnitude of the two stretching compliances $k_{s} / k_{\|} \simeq R^{2}\left(l / l_{p}\right)^{3}$.

The dependence of the three force constants $k_{\perp}, k_{s}$, and $k_{\|}$, Eqs. (3) and (4), on the ratio of persistence length to segment length $l_{p} / l$ is illustrated in Fig. 3. One can clearly distinguish three regimes, in each of which one of the spring constants is by far smaller than the remaining two. The dashed line corresponds to a hypothetical spring where the deformation modes are coupled in series $k^{-1}=k_{\perp}^{-1}+k_{s}^{-1}+k_{\|}^{-1}$. If the segment length $l$ were representative for the network under consideration - that is, the network is characterized by only small polydispersity-then we would expect the macroscopic modulus to be well approximated by the microscopic single-segment behavior considered here. We will later refer to this behavior as the "affine model." It will be shown to be valid only in regular cellular structures.

This completes the specification on the microscopic scale of the elastic properties of the single polymer segments. We

\footnotetext{
${ }^{1}$ This limit corresponds to taking $r \rightarrow 0$ and the Youngs modulus $E \rightarrow \infty$ in such a way that the bending stiffness $\kappa \sim E r^{4}$ stays constant. As a consequence the stretching stiffness diverges, $k_{s} \sim E r^{2}$ $\rightarrow \infty$, and the beam becomes inextensible.
} 
now proceed to assemble the segments into networks of varying architecture to identify the physical principles which determine the elastic response on the macroscopic scale.

To determine the elastic shear modulus, we apply a small shear strain of $\gamma=0.01$ to stay in the regime of linear elasticity and use periodic boundary conditions on all four sides of the simulation box. The numerical procedure is performed with the commercially available finite-element solver MSC.MARC. The results will be complemented by scaling arguments.

We will find that in regular cellular architectures, to be discussed next, macroscopic elasticity can trivially be explained by the microscopic constitutive laws given in terms of the stiffness matrix $\mathbf{K}$. In sufficiently random cellular systems, however, this picture is changed. The macroscopic response takes up nontrivial features that cannot be explained by single polymer elasticity. In fibrous architectures, the subject of Sec. IV, we will find this anomalous elasticity again but in more striking form.

\section{CELLULAR ARCHITECTURE}

A cellular structure is most conveniently constructed from a Voronoi tessellation of a distribution of points which may either be chosen regularly or by some random process [29]. With each point we associate a Voronoi cell that is defined to enclose that region in space which is closer to the given point than to any of its neighbors. This procedure is equivalent to the Wigner-Seitz construction known from solid-state physics. In three dimensions the elastic elements are defined to be the lines of intersection of two neighboring cell walls, while in two dimensions (see Fig. 1) they are represented by the cell walls themselves. We will call these elastic building blocks of the network polymer segments and associate to them the material properties, respectively the stiffness matrix $\mathbf{K}$, introduced in the preceding section. By its definition, a segment spans the distance between two vertices and is therefore "end-linked" to the rest of the network.

Depending on the spatial distribution of Voronoi points there will also be a distribution $P\left(l_{s}\right)$ of segment lengths $l_{s}$. Only in regular structures-for example, the (anisotropic) two-dimensional honeycomb structure-will this distribution degenerate into one (or several) $\delta$-function peaks.

The first moment of this distribution, the average segment length $\bar{l}_{s}$, is naturally the most important quantity to describe the geometrical aspects of a cellular structure. In $d=2,3$ dimensions this "mesh size" may be reparametrized in terms of the density $\rho$ as

$$
\bar{l}_{s} \propto \rho^{-1 /(d-1)},
$$

where we defined $\rho$ as the total polymer length per system size. While there are practical reasons to use $\rho$ as a measure for the density in the simulations, in experimental work it is sometimes easier to control the monomer concentration $c$. This can be found as $r c \propto \rho$, where the cross-section radius $r$ is assumed proportional to the monomer size.

\section{A. Mechanical behavior: Beams}

In the engineering literature the cellular structures defined above are well known as foams and are ubiquitous in nature and many areas of technology. Examples range from liquid foams and froths, well known from drinks or household detergents, to plastic and metallic foams used for insulation or shock absorption $[29,30]$. It is well known that naturally occurring foams have to obey Plateau's laws to reach an equilibrium state. We do not require these laws to hold in the following, since we are interested in the dependence of elastic properties on the architectural features in general and not in the specific details of the dynamic properties of foams.

For purely mechanical cellular foams, where thermal fluctuations are neglected altogether, the only material length scale is the radius $r$ of the cross section. By identifying $\kappa / \bar{l}_{s}$ as an energy scale, we can use dimensional analysis to write the shear modulus $G$ as

$$
G=\frac{\kappa}{\bar{l}_{s}^{d+1}} g\left(r / \bar{l}_{s}\right),
$$

where the occurrence of the spatial dimension $d$ highlights the fact that the modulus has units of an energy density. In writing this, we have not made explicit the dependence on the higher moments of the probability distribution $P$. As will become clear below, these can be used to characterize the randomness of the structure and will be considered separately. If one defines force constants at the scale of the average mesh size,

$$
\bar{k}_{\perp} \simeq \kappa / \bar{l}_{s}^{3}, \quad \bar{k}_{s} \simeq \kappa / \bar{l}_{s} r^{2},
$$

the scaling variable can alternatively be written as $r / \bar{l}_{s}$ $\simeq \sqrt{\bar{k}_{\perp} / \bar{k}_{s}}$ and, therefore, characterizes the relative stiffness of the bending to the stretching mode.

\section{B. Regular structures and affine models}

Restricting our attention for the moment to regular structures, macroscopic elasticity can already be understood by considering the response of a single cell [30-32]. In these systems it seems reasonable that local stresses acting on an individual cell are the same as those applied on the macroscopic scale. In other words, the local deformation $\delta$ of a cell with linear dimension $\bar{l}_{s}$ follows the macroscopic strain $\gamma$ in an affine way such that it scales as $\delta \propto \gamma \bar{l}_{s}$. With this assumption the scaling function can be calculated [31] and one generically finds for the modulus

$$
G_{\mathrm{aff}}^{-1}=\bar{l}_{s}^{d-2}\left(a \bar{k}_{\perp}^{-1}+b \bar{k}_{s}^{-1}\right),
$$

where the details of the particular structure may enter the numbers $a$ and $b$ in an involved way. The important conclusion to be drawn is that the deformation modes act as if they were springs connected in series. For slender beams with $r$ $\ll \bar{l}_{s}$ the bending mode is softer than the stretching mode and therefore dominates the modulus-mechanical foams are bending dominated.

While we argue here that the modulus in Eq. (9) represents the generic case, there may be special cases were the 
prefactors $a$ are $b$ are suppressed by the specific choice of the unit cell. The triangulated network is one example where $a$ $=0$ and the bending mode is suppressed. Below we will encounter another example when studying the square lattice. For these systems the special geometry of the unit cell, or more generally the local architecture, has to be taken into account. This is indeed the main focus of this article. On the other hand, by assuming affine displacements no cooperativity between the elastic responses of neighboring cells is possible. The macroscopic modulus $G$ directly reflects the elastic properties of the single cell. The local geometry is being hidden in the prefactors $a$ and $b$, while the effect of the assembled structure may simply be predicted by counting the numbers of cells.

\section{Cell polydispersity}

We have tested the validity of the affine model in a simple two-dimensional (2D) cellular structure with varying degree of randomness. We have taken the seeds for a Voronoi construction of a regular, honeycomb lattice structure and randomly displaced them with a uniform probability distribution of width $\Delta \times \bar{l}_{s}$. The influence of randomness on the elastic properties of mechanical (nonfluctuating) foams has been studied extensively by various authors [33-35]. Here, we also include effects from thermal fluctuations such that the response of a polymer segment is characterized by three deformation modes with stiffnesses $k_{s}, k_{\|}$, and $k_{\perp}$, respectively. The affine prediction for the modulus of this system $(d=2)$ can be inferred from Eq. (9). By defining

$$
\bar{k}_{\|} \simeq \kappa l_{p} / \bar{l}_{s}^{4}
$$

and substituting $\bar{k}_{s}^{-1} \rightarrow \bar{k}_{s}^{-1}+\bar{k}_{\|}^{-1}$, one finds for the modulus

$$
G_{\mathrm{aff}}^{-1}=\bar{k}_{\perp}^{-1} h\left(l_{p} / \bar{l}_{s}\right)=\frac{\bar{l}_{s}^{3}}{\kappa}\left[a+b\left(\frac{R \bar{l}_{s}}{l_{p}}\right)^{-2}+c \frac{\bar{l}_{s}}{l_{p}}\right],
$$

where we have inserted Eqs. (8) and (10) and used the relation $R=l_{p} / r$. This has to be compared with the actual results of our numerical analysis in Fig. 4. The normalized shear modulus $G \overrightarrow{l_{s}^{3}} / \kappa$ is shown as a function of persistence length $l_{p} / \bar{l}_{s}$ expressed in units of the average segment length. The curves correspond to varying degrees of randomness $\Delta$.

We find that regular networks (black curve, circles) characterized by a single mesh size $\bar{l}_{s}$ indeed display the functional form expressed through Eq. (11). For mesh sizes much larger than the persistence length $\bar{l}_{s} \gg l_{p}$ the network deforms by pulling out thermal undulations and $G \propto \bar{k}_{\perp} \propto \bar{l}_{s}^{-4}$ (left part of Fig. 4). Decreasing the mesh size beyond $\bar{l}_{s} \approx r$ stretching of the polymer backbone dominates the modulus $G \propto \bar{k}_{s}$ $\propto \bar{l}_{s}^{-1}$ (right part of Fig. 4). The physically relevant situation for studying stiff polymers, however, corresponds to the intermediate regime, where the persistence length is much larger than the mesh size, which is still much larger than the polymer radius $l_{p} \gg \bar{l}_{s} \gg r$. Typical actin networks with $l_{p}$ $=17 \mu \mathrm{m}$ and $r=5 \mathrm{~nm}$ may have mesh sizes in the submicron range $\bar{l}_{s} \approx 100 \mathrm{~nm}$. In this regime, most of the energy is

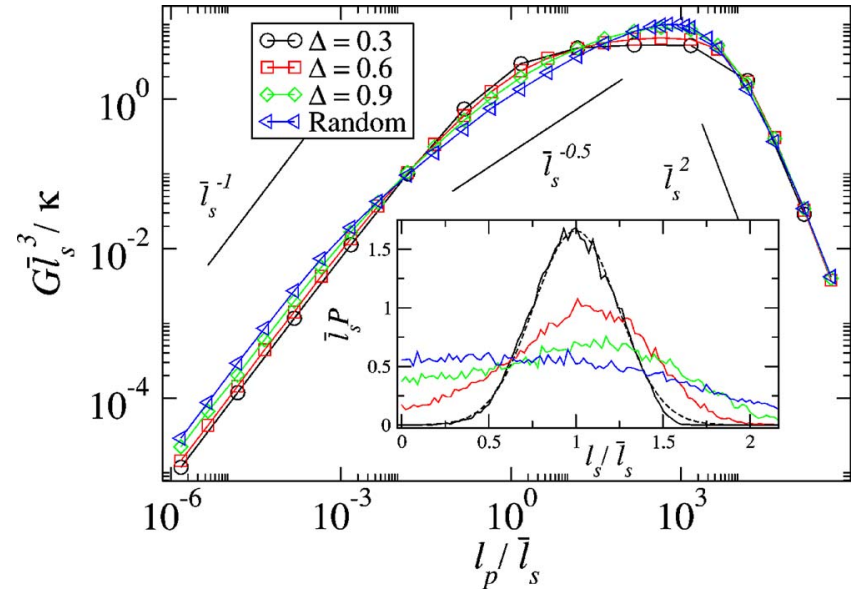

FIG. 4. (Color online) Shear modulus $G \overrightarrow{l_{s}^{3}} / \kappa$ as a function of $l_{p} / \bar{l}_{s}$ for a $2 \mathrm{D}$ honeycomb foam structure with varying degree of randomness $\Delta$. The blue curve ("Random") corresponds to a "maximally" random foam generated from a Poisson point process. Inset: distribution $P$ of segment lengths for the same systems. At low levels of randomness $(\Delta=0.3)$ it can be approximated by a Gaussian probability distribution (dashed line), while it shows significant broadening upon increasing the randomness to $\Delta=0.6,0.9$. The peak disappears completely in the case of the maximally random Poisson foam.

stored in the bending modes leading to $G \propto \bar{k}_{\perp} \propto \bar{l}_{s}^{-3}$ corresponding to the plateau region visible in Fig. 4.

Using the values $a=0.2, b=0.35$, and $c=0.14$ we managed to fit the scaling function of Eq. (11) to the numerical data (in fact, this is the dashed line in Fig. 3). Increasing the level of randomness the presence of the additional variable $\Delta$ spoils the scaling property and a fit is no longer possible. The power-law regimes gradually shrink and the crossover regions increase in size. While the mechanical stretching regime is hardly affected by the randomness at all, this effect is most pronounced in the crossover from the bending to the thermal stretching dominated regime. The physically most relevant intermediate plateau regime disappears completely and shows strong amplitude modulations.

We have also generated foams by Voronoi tessellation of a fully random distribution of points, corresponding to a Poisson process (blue curve, left triangles). For these "maximally random foams" one could rather use an expression $G \propto \bar{l}_{s}^{-7 / 2}$ to characterize the modulus at these intermediate parameter values. At this point this is only an empirical observation. Later, in the context of the fibrous architecture, we will see how this exponent can be derived from a scaling argument that properly takes into account the randomness in the system.

One may infer from the inset of Fig. 4 that deviations from the scaling form presented in Eq. (11) are indeed intimately connected to a broadening of the segment length distribution $P\left(l_{s}\right)$. In the regular structure the distribution can very well be described by a Gaussian centered around the average mesh size $\bar{l}_{s}$ (dashed line in Fig. 4). Random foams, on the contrary, display significantly broader distributions and even have non-negligible weight on very small segments. 

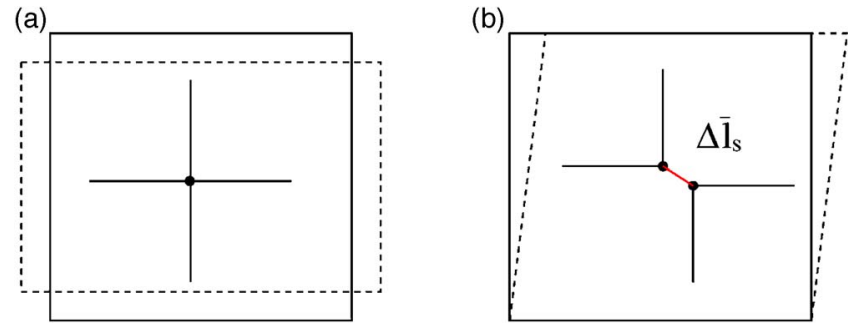

FIG. 5. (Color online) (a) Pure shear deformation of the square lattice and (b) simple shear. Illustration of the bifurcation leading from a fourfold connected vertex to a threefold connected one by introducing small amounts of randomness $\Delta \bar{l}_{s}$.

We will see below that the different effects of randomness in the thermal and the mechanical stretching regimes can be traced back to the unusually strong length dependence of the entropic stretching stiffness, $k_{\|} \propto l_{s}^{-4}$, as compared to $k_{s} \propto l_{s}^{-1}$. We will find that this leads to the breakdown of the affine model whenever there is a sufficiently broad distribution of segment lengths. Thermal networks are thus inherently more sensitive to elements of randomness than purely mechanical systems.

It is instructive to consider yet another lattice structure as a basis for our foam model (see Fig. 1). By placing the Voronoi points on a slightly randomized square lattice one can generate a foam with a bimodal segment length distribution having a second peak at some small length $l_{1}$ (see inset, Fig. 6). To understand this, one has to realize that a generic foam structure generated by Voronoi tessellation has only threefold connected vertices, while they are fourfold connected in the square network. A small amount of randomness therefore induces a bifurcation of a fourfold vertex into a short segment with threefold connected vertices at its ends (see Fig. 5). Unlike the honeycomb foam, the resulting structure is elastically anisotropic and has three distinct moduli [36]. In addition to the bulk modulus there are two shear moduli corresponding to simple and pure shear deformations. These two modes are schematized in Fig. 5, while the corresponding moduli (together with the isotropic shear modulus of the Poisson foam) are shown in Fig. 6. Pure shear leads to deformations along the main axis of symmetry of the unit squares and thus to stretching of the elements. The bending regime is therefore strongly suppressed. On the other hand, simple shear deforms the squares along their diagonals and thus favors the bending mode. Only when the stretching energy stored in the small segments $w_{\|}$ $=k_{\|}\left(l_{1}\right) \delta_{\text {aff }}\left(l_{1}\right)^{2} \propto l_{p} / l_{1}^{2}$ equals the bending energy in the average segment $w_{\perp}=k_{\perp}\left(\bar{l}_{s}\right) \delta_{\text {aff }}\left(\bar{l}_{s}\right)^{2} \propto \bar{l}_{s}^{-1}$ does the system cross over to a stretching dominated network. Noting (from the inset of Fig. 6) that $l_{1} \approx \bar{l}_{s} / 10$ we find that this happens when $l_{p} \approx 10^{-2} \bar{l}_{s}$ in accordance with Fig. 6 . It is interesting to see that the network loses its anisotropy at the two points $\bar{l}_{s}=l_{p}$ and $\bar{l}_{s}=r$, where the modulus takes the same value as that of the Poisson foam. This follows from the fact that the stiffness of the average polymer segments is isotropic at these parameter values and either $\bar{k}_{\|} \approx \bar{k}_{\perp}$ or $\bar{k}_{s} \approx \bar{k}_{\perp}$. Comparing absolute values we find that the shear modulus in the thermal regime,

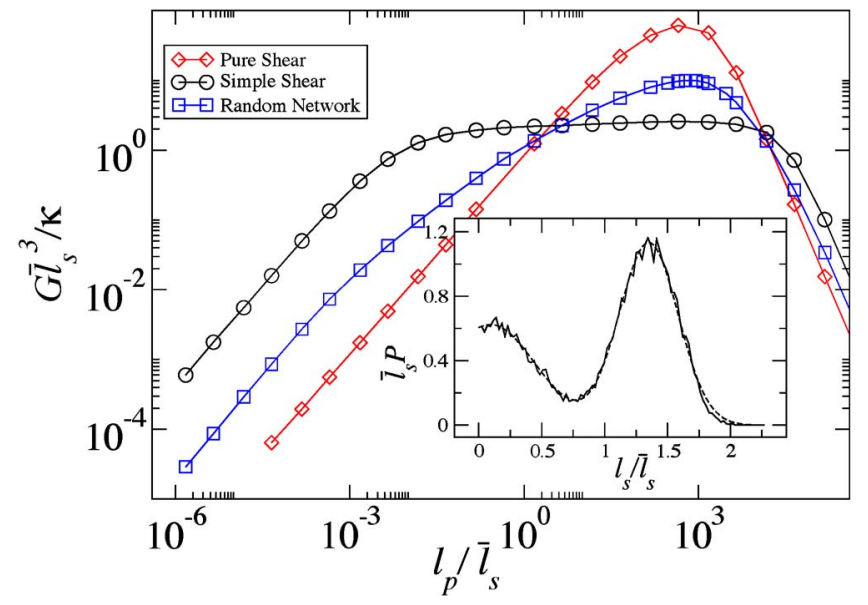

FIG. 6. (Color online) The two different shear moduli for the slightly randomized square lattice $(\Delta=0.3)$ as shown in Fig. 1. Also shown is the modulus of the highly random Poisson foam. Inset: distribution of segment lengths for the same network. The dashed line is a fit to a sum of two Gaussians centered around $l_{1} / \bar{l}_{s}$ $=0.092$ and $l_{2} / \bar{l}_{s}=1.35$.

strongly influenced by the presence of the small segments, can vary orders of magnitude while the mechanical stretching regime is hardly affected at all.

To conclude this section we emphasize once again that polydispersity in the segment lengths can have strong effects on the macroscopic elasticity of a cellular polymer network. It can lead to modifications of the scaling properties, as we have found in the most random foams, as well as to quantitative changes of the modulus by several orders of magnitude as in the anisotropic square structure. As a consequence, experiments which are limited to restricted parameter windows would most likely measure effective exponents that lie in between the extremal values given by pure stretching and bending. One therefore has to be cautious interpreting experimental data within the context of the foam model without knowledge of the polydispersity of the structure.

\section{FIBROUS ARCHITECTURE}

Looking at pictures of cross-linked actin networks reconstituted in vitro $[12,15]$ one might wonder whether a description in terms of a cellular architecture is actually relevant for these systems at all. Besides having a strong polydispersity in cell sizes, real polymer networks seem to have a hierarchical architecture that allows for smaller cells to be generated within larger cells within even larger cells. On the contrary, foams only have one of these hierarchies (see Fig. 1). What is more, cellular structures do not account for the effects of the polymer length $l_{f}$, which constitutes an additional mesoscopic scale in the problem.

In the following we want to quantify the effects of the polydispersity in connection with the length scale $l_{f}$ by studying the elastic properties of a generic two-dimensional fibrous structure which is defined as follows. $N$ anisotropic elastic elements, geometrically represented by straight lines of length $l_{f}$, are placed on a plane of area $A=L^{2}$ such that 


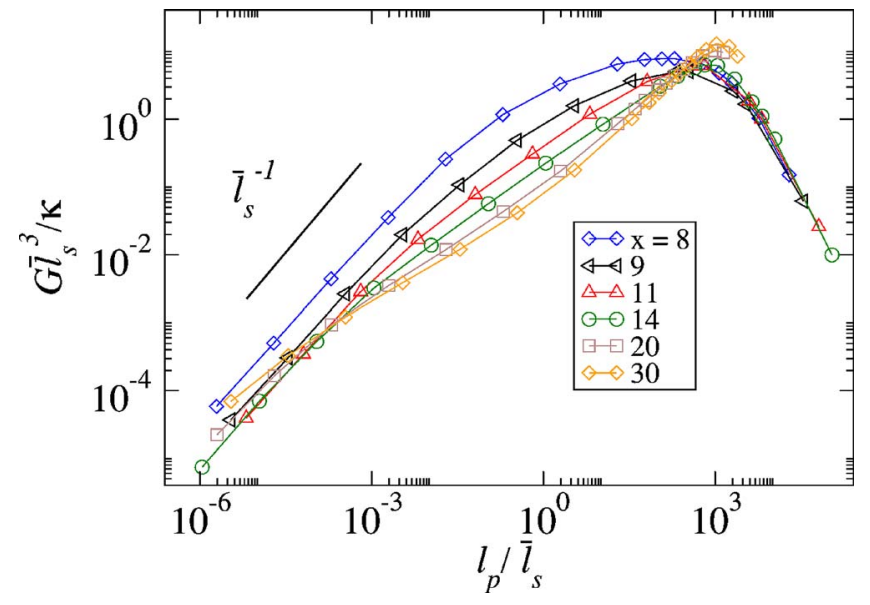

FIG. 7. (Color online) Scaling function $g$ as a function of $y$ $=l_{p} / \bar{l}_{s}$ for various $x=l_{f} / \bar{l}_{s} \leqslant 30$ (from networks with freely hinged cross-links). For rather small values of $x=8,9$ the curves resemble the results from the cellular networks. At intermediate values $10^{-3}$ $\leqslant y \leqslant 10^{2}$ the modulus shows strong modulation and develops a dip with increasing $x$.

both position and orientation of the elements are uniformly random distributed. This randomness entails a distribution of angles $\theta \epsilon[0, \pi]$ between two intersecting filaments,

$$
P(\theta)=\frac{\sin (\theta)}{2},
$$

which has a maximum for filaments at right angles. At any intersection a permanent cross-link with zero extensibility is generated. This constrains the relative translational motion of the two filaments. For the rotational degree of freedom one may introduce an energy contribution $W_{\text {rot }}=m\left(\phi-\phi_{0}\right)^{2}$ for the change of relative cross-link angles $\phi$ from their initial values $\phi_{0}$. We restrict ourselves to the study of the two limiting cases, where the potential is either soft $(m \rightarrow 0)$, and therefore allows for free relative rotations of the filaments (free hinges), or infinitely stiff $(m \rightarrow \infty)$ and inhibits any change of the angles at the cross-links (fixed angles).

The remaining elastic building blocks of the network, the polymer segments, span the distance between two neighboring cross-links on the same polymer. Their length can be shown to follow an exponential distribution [37]

$$
P\left(l_{s}\right)=\bar{l}_{s}^{-1} e^{-l_{s} \bar{l}_{s}} .
$$

The mean value $\bar{l}_{s}$ is given in terms of the density $\rho=N l / A$ as

$$
\bar{l}_{s}=\pi / 2 \rho,
$$

which is a realization of Eq. (6). On average there are, thus, $x=l_{f} / \bar{l}_{s} \approx l_{f} \rho$ segments per polymer. The simplicity of this network, which has only one structural parameter $\rho$, makes it an ideal candidate to obtain physical insight into the relation between architecture and elastic properties of the constituents. This model has frequently been used to study the elastic and brittle properties of athermal paper sheets [25,38-40]. In the context of biological networks of stiff polymers it has

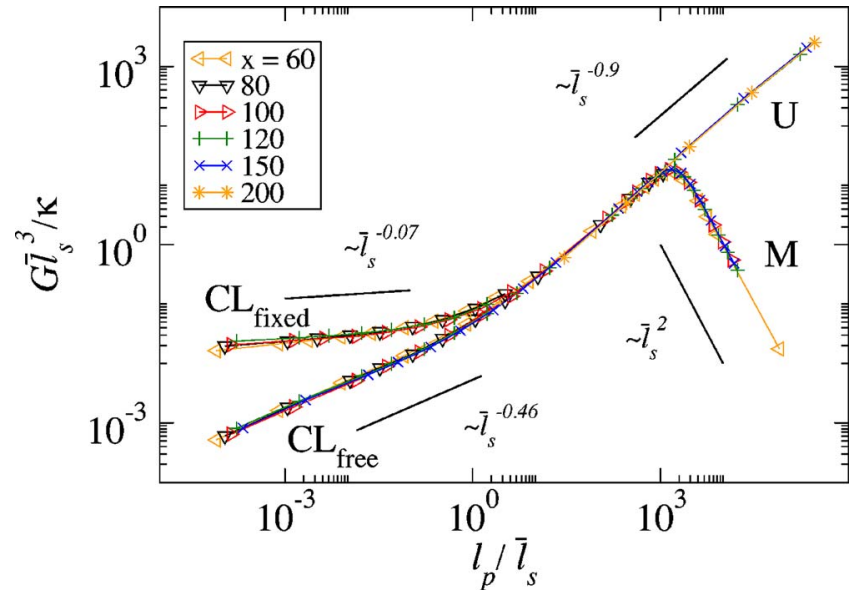

FIG. 8. (Color online) Scaling function $g$ as a function of $y$ $=l_{p} / \bar{l}_{s}$ for various $x=l_{f} / \bar{l}_{s} \geqslant 60$. In contrast to Fig. 7 no dependence on $x$ is observed anymore and new scaling regimes emerge. The two branches in the cross-link-dominated regime $(y \ll 1)$ correspond to freely hinged $\left(\mathrm{CL}_{\text {free }}\right)$ or fixed $\left(\mathrm{CL}_{\text {fixed }}\right)$ cross-link angles, respectively. For $y \gg 1$ one encounters a universal thermal regime $(U)$ independent of the cross-link properties as well as a mechanical regime $(M)$.

been introduced in [24] and recently studied by various authors [19-21]. In all this work, however, the elastic properties of the polymers are modeled by the classical theory of Euler-Bernoulli beams. Here, we concentrate on the effects of thermal fluctuations, a brief account of which we have published recently [23].

\section{A. Simulation results}

In Figs. 7 and 8 the results of our simulations are shown for fibrous networks with a varying number $x$ of cross-links per polymer. The axes are the same as in previous plots. The normalized shear modulus $G \overline{l_{s}^{3}} / \kappa$ is shown as a function of persistence length $y=l_{p} / \bar{l}_{s}$ expressed in units of the average segment length. Short fibers with few cross-links, corresponding to low densities, are depicted in Fig. 7, long fibers or high densities in Fig. 8. In both figures we find a regime at large values of the persistence length $l_{p} / \bar{l}_{s}$ (right part of the plot) where the dimensionless shear modulus decreases as $G \propto l_{p}^{-2} \propto r^{-2}$. This corresponds to a purely mechanical stretching regime where $G \propto \bar{k}_{s}$ consistent with the mean-field picture of Eq. (11) $[19,20,24,25]$.

Our main interest, however, lies in the regime of $l_{p} / \bar{l}_{s}$ $\leqslant 10^{3}$, where the persistence length is small enough for thermal fluctuations to become relevant. In this regime one may safely neglect the mechanical stretching stiffness and set $k_{s}$ $\rightarrow \infty$. Then, dimensional analysis for the shear modulus requires

$$
G=\frac{\kappa}{\vec{l}_{s}^{3}} g(x, y),
$$

where we have introduced the scaling variables 


$$
x=l_{f} / \bar{l}_{s} \simeq l_{f} \rho, \quad y=l_{p} / \bar{l}_{s} \simeq l_{p} \rho .
$$

Comparing to Eq. (7) there is an additional argument in the scaling function $g$, the polymer length $x=l_{f} / \bar{l}_{s}$. This purely geometrical variable counts the number of cross-links (or, equivalently, segments) per filament. The second argument may be written in the alternative form $y \simeq \bar{k}_{\|} / \bar{k}_{\perp}$. It characterizes the relative stiffness of stretching and bending mode for a typical segment.

From Fig. 7 one infers that for low densities $g=y f(x)$, implying for the modulus $G=\bar{k}_{\|} f\left(\rho l_{f}\right)$. This linear dependence on the "preaveraged" stretching compliance $\bar{k}_{\|}$hints at an entropic stretching-dominated regime similar to that found in the cellular structures discussed above. This regime has been suggested in [21], where a scaling argument is developed relying on the affine assumption borrowed from the mechanical stretching regime. Our analysis shows that the domain of validity of this linear regime is extremely narrow and confined to short filaments $x \leqslant 20$ and persistence lengths $y \ll 1$. What is more, due to the nontrivial density dependence expressed through the function $f(x)$, the modulus does not even display a power-law behavior in the density. Instead, we find that the modulus shows complex dependence on its variables and develops a dip in the intermediate parameter region where $10^{-3} \leqslant y \leqslant 10^{2}$. This is also the relevant parameter range for networks of F-actin, where the ratio of persistence length to mesh size $l_{p} / \bar{l}_{s} \approx 10-100$.

For medium and high densities Fig. 8 shows nontrivial scaling regimes where the scaling function $g$ becomes independent of $x$ and therefore of the filament length $l_{f}$. This highly nontrivial observation has important implications and allows the system to exhibit power law behavior $g \propto y^{z}$. We find nontrivial fractional exponents $z=0.46(0.07)$ and $z=0.9$ for small and large values of $y$, respectively. In the figure one can distinguish four branches that belong to different realizations of the network. While branch $M$ (mechanical regime, $G \propto \bar{k}_{s}$ ) has been discussed already, the remaining three are obtained by setting $k_{s} \rightarrow \infty$. The two branches found at small values $y \ll 1$ relate to networks where the cross-link angles are either free to vary $\left(\mathrm{CL}_{\text {free }}, z=0.46\right)$ or are perfectly fixed to their initial values $\left(\mathrm{CL}_{\text {fixed }}, z=0.07\right)$, respectively. We term this regime "cross-link dominated" since tuning the crosslink properties may have strong effects on the elastic modulus by driving the system from one branch towards the other. Both branches merge at $y \approx 1$ where we enter a universal regime (branch $U, z=0.9$ ) which is completely independent of the elasticity of the cross-links and which therefore is termed "filament dominated."

In all cases, the modulus can be written as a generalized geometric average

$$
G \propto \bar{k}_{\perp}^{1-z} \overline{k_{\|}^{z}},
$$

which has to be contrasted with Eq. (11), where bending and stretching modes are assumed to superimpose linearly (see Table I for a direct comparison of the various regimes). There, the system is described either by $z=0$, if bending dominates, or by $z=1$, if stretching is the main deformation
TABLE I. Compilation of the different elastic regimes of the fibrous network. The modulus is given by $G \sim \bar{k}_{\perp}^{1-z} \bar{k}_{\|}^{z}$ with the appropriate values for the exponent $z$. For comparison also the predictions from the theoretical analysis (see below) as well as the exponents for the foam structure are given, the latter only for fixed cross-link angles $\left(\mathrm{CL}_{\text {fixed }}\right)$, which is necessary to make the structure stable. The mechanical regime $M$ corresponds to the exponent $z$ $=1$, however with $\bar{k}_{\|}$substituted by $\bar{k}_{s}$.

\begin{tabular}{llccc}
\hline \hline & & $z$ & $z_{\text {theory }}$ & $z_{\text {foam }}$ \\
\hline $\mathrm{CL}_{\text {fixed }}$ & $r \ll l_{p} \ll \bar{l}_{s}$ & 0.07 & 0 & 1 \\
$\mathrm{CL}_{\text {free }}$ & $r \ll l_{p} \ll \bar{l}_{s}$ & 0.46 & $1 / 2$ & - \\
$U$ & $r \ll \bar{l}_{s} \ll l_{p}$ & 0.9 & 1 & 0 \\
$M$ & $\bar{l}_{s} \ll r \ll l_{p}$ & $(1)$ & $(1)$ & $(1)$ \\
\hline \hline
\end{tabular}

mode. Values different from the two limiting cases $z=0,1$ cannot be described by the mean-field approach; hence, the assumption of affine deformations applied on the level of the polymer segments (or the cell size) necessarily has to fail. This will become especially clear in the following section, where we review the application of affine theories to fibrous architectures. We will illustrate its failure and highlight the physical principles involved. To go beyond we will introduce a model that accounts for the spatial distribution of crosslinks along the backbone of a typical polymer filament instead of just considering a single typical polymer segment. This approach will allow us to understand all the features of the macroscopic elasticity encountered in Fig. 8.

\section{B. Affine models in fibrous architectures}

In some of the earlier approaches to describe the elastic moduli of stiff polymer networks the assumption of affine deformations has been applied on the level of the average segment which can be characterized by "preaveraged" response coefficients $\left\langle k\left(l_{s}\right)\right\rangle \rightarrow \bar{k}=k\left(\bar{l}_{s}\right)$ introduced in Eqs. (8) and (10). The characteristic fibrous structure of stiff polymer networks is not accounted for and effectively substituted by a highly regular cellular structure. The modulus in the thermal regime is then obtained simply by replacing in Eq. (9) the mechanical stretching response $\bar{k}_{s}$ with its thermal counterpart $\bar{k}_{\|}$. Several variants of this model have been considered in the literature $[27,28,41]$, which only differ in the specific (ad hoc) choice of the prefactors $a$ and $b$. The stretchingdominated model [28] [setting $a=0$ in Eq. (9)] with a modulus depending on density as

$$
G_{\|} \sim \rho^{(2+d) /(d-1)}
$$

and its extensions to nonlinear elasticity [42] have widely been used to fit experimental data for the plateau modulus in cross-linked F-actin networks $[12,15,43]$. Despite this apparent success, it is not clear a priori why in the parameter regime of interest the mesh work should deform by the stretching of bonds when actually bending is by far the softer mode $\left(\bar{k}_{\|} / \bar{k}_{\perp} \simeq l_{p} / \bar{l}_{s} \gg 1\right)$. In general, such a regime can only 


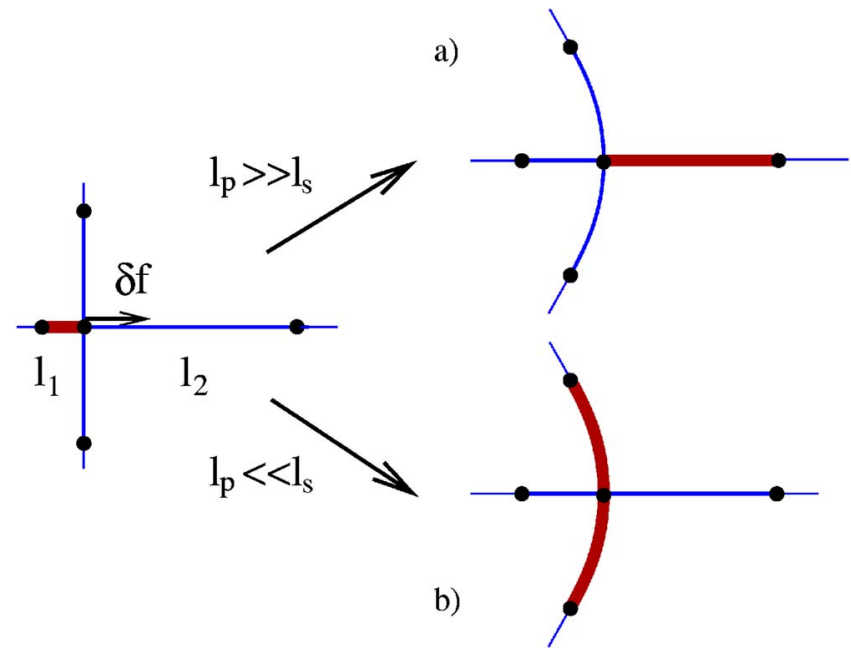

FIG. 9. (Color online) Illustration of the effects of nonzero residual forces. The relaxation of the small segment $l_{1}$ from its overly stressed state goes to the cost of additional deformations in its neighbors. Depending on the value of the persistence length the energy will mainly be stored in (a) the stretching mode $\left(l_{p} \gg \bar{l}_{s}\right)$ or (b) the bending mode $\left(l_{p} \ll \bar{l}_{s}\right)$.

occur if the specific architecture suppresses the soft bending modes as in the triangulated structure with its highly coordinated vertices. A second approach seems to repair this deficiency by setting in Eq. (9), $b=0$. The modulus in this theory,

$$
G_{\perp} \sim \rho^{(1+d) /(d-1)},
$$

only differs by a factor of $\rho^{1 /(d-1)}$ from the stretchingdominated modulus of Eq. (18). However, neither theory provides justification for neglecting the effects of the polydispersity in the fibrous system. In fact, if one extends the approach to include the distribution of segment lengths, such theories necessarily have to fail, as we will explain in the next section.

\section{Effects of the segment length distribution}

To understand the origin of this failure consider an affine deformation field $\delta_{\text {aff }} \propto \gamma l_{s}$ being applied to a random network of stiff polymers with a distribution $P\left(l_{s}\right)$ of segment lengths $l_{s}$. The axial forces $f_{\|}$generated by such a deformation field can simply be calculated by multiplying the deformation with the stretching stiffness of the segment:

$$
f_{\|}=k_{\|} \delta_{\mathrm{aff}} \simeq \kappa l_{p} \gamma / l_{s}^{3} .
$$

Note that in contrast to the purely mechanical situation, where the axial force $f_{s}=k_{s} \delta_{\mathrm{aff}} \simeq \kappa \gamma / r^{2}$ is independent of length, $f_{\|}$strongly increases with shortening the segment length. This implies that, in general, two neighboring segments on the same filament produce a net force $\delta f$ at their common node that has to be taken up by the crossing filament. There, it leads to additional deformations that eventually destroy the affine order. This mechanism is illustrated in Fig. 9 where the relaxation of the small segment $l_{1}$ leads to bending of its neighbor on the crossing filament (b). Also the segment $l_{2}$ on the same filament is affected by the relaxation and experiences an additional stretching contribution (a). Whether the available energy is stored in the stretching or the bending mode depends crucially on the value of the persistence length, as is indicated in the figure.

One may calculate the probability distribution $Q$ for residual forces by summing over all segment lengths that are consistent with a given force $\delta f$,

$$
Q(\delta f)=\left\langle\delta\left(\left|f_{\|}\left(l_{1}\right)-f_{\|}\left(l_{2}\right)\right|-\delta f\right)\right\rangle .
$$

The averaging procedure, defined by the angular brackets

$$
\langle A\rangle=\int d l_{1} \int d l_{2} P\left(l_{1}, l_{2}\right) A\left(l_{1}, l_{2}\right),
$$

involves the two-point probability $P\left(l_{1}, l_{2}\right)$ of finding neighboring segments with lengths $l_{1}$ and $l_{2}$, respectively. In the special case of the random network considered here, there are no correlations between neighboring segment lengths such that the distribution factorizes. The formula can thus be evaluated by substituting $f_{\|} \propto l_{s}^{-3}$ taken from Eq. (20). This inverse relationship between forces and segment lengths translates the weight of the probability distribution $P_{0}=P\left(l_{s}\right.$ $\rightarrow 0) \neq 0$ at small segment lengths into polynomial (fat) tails of the corresponding distribution of residual forces,

$$
Q(\delta f \rightarrow \infty) \propto \delta f^{-4 / 3} P_{0},
$$

which has a diverging mean value. The exponent can readily be derived from evaluating the integral measure $d f_{\|} \propto l^{-4} d l$ $\propto f_{\|}^{4 / 3} d l$. As a consequence there are always residual forces high enough to cause additional deformation of the crossing filament. Hence we conclude that an affine deformation field is unstable and that the system can easily lower its energy by redistributing the stresses to relieve shorter segments and remove the tails of the residual force distribution $Q(\delta f)$.

Even though we have evaluated Eq. (21) for the special case of an exponential segment length distribution, Eq. (13), it is important to note that the observed sensitivity is not a special feature of the fibrous architecture, but applies to any polymer network with a broad distribution of segment lengths independent of the dimensionality of the network. Due to the strong length dependence of $k_{\|}\left(l_{s}\right)$, the thermal response is highly sensitive to even small polydispersity as we have already seen in the random cellular network of Sec. III C. On the contrary, these effects are completely absent in purely mechanical models and also in models of flexible polymers, where the distribution $Q(\delta f)$ degenerates into a delta-function peak at the value $\delta f=0$ and explains the robustness of these regimes to randomness.

If we want to include the effects of randomness into a microscopic theory, we cannot naively apply the conventional picture of affine deformations on the scale of the single segment. This can safely be done only in highly ordered structures like regular cellular materials. Instead, we have to adopt a description of the deformations (at least) on the larger scale of the complete polymer. In the following we therefore consider a typical polymer filament, which is composed of a sequence of segments drawn from the distribution $P\left(l_{s}\right)$. To describe the elastic properties correctly, we will 


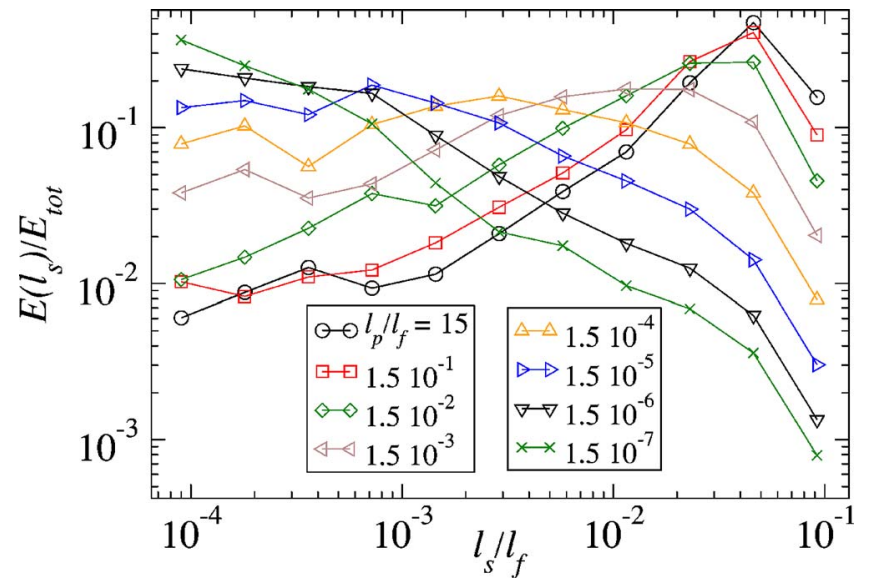

FIG. 10. (Color online) Fraction of energy stored in the various segment lengths; the curves correspond to different persistence lengths at a density of $l_{f} / \bar{l}_{s}=80$, equivalent to $\bar{l}_{s} / l \approx 2 \times 10^{-2}$.

also have to consider the connections of the polymer to the surrounding network matrix, in addition to the elastic properties of the segments themselves. We may now employ this picture to explain the intricate scaling properties of the polymer network in all the parameter regimes displayed in Fig. 8.

\section{Cross-link-dominated regime}

\section{Freely hinged cross-links}

We start with the description of the system in the parameter region $y \ll 1\left(l_{p} \ll \bar{l}_{s}\right)$, where the properties of the crosslinks strongly influence the system's response. The idea is to impose a virtual affine deformation on every segment and calculate, as a perturbative correction, the contribution to the elastic energy resulting from the relaxation out of this reference state. This procedure will lead to good predictions only when the corrections are small and the affine deformations are only weakly perturbed. As we will see below, this is the case in the parameter region $y \ll 1$. However, it will also become clear that a small perturbation for the deformations is sufficient to generate completely different scaling properties for the macroscopic modulus. For the moment we restrict our attention to free relative cross-link rotations (branch $\mathrm{CL}_{\text {fixed }}$ ), since then the affine reference state is particularly simple and contains stretching contributions only.

As explained above any deviation from the affine reference state, induced by relaxation of nonzero residual forces, will lead to additional deformations in the crossing filaments. Since it is more likely that two filaments cross each other at an angle close to $90^{\circ}$, the induced nonaffine deformations will mainly be oriented transverse to the contour of the crossing filament and are therefore of bending character. The value of the exponent $z=0.46$ supports this assumption and indicates that bending and stretching deformations in this regime contribute equally to the elastic energy even though the bending mode is very stiff $\left(\bar{k}_{\|} / \bar{k}_{\perp} \sim y \ll 1\right)$. Therefore any relaxation of residual stretching forces will be punished by high amounts of bending energy [see Fig. 9(b)]. Only the smallest segments on the polymer, corresponding to the out-

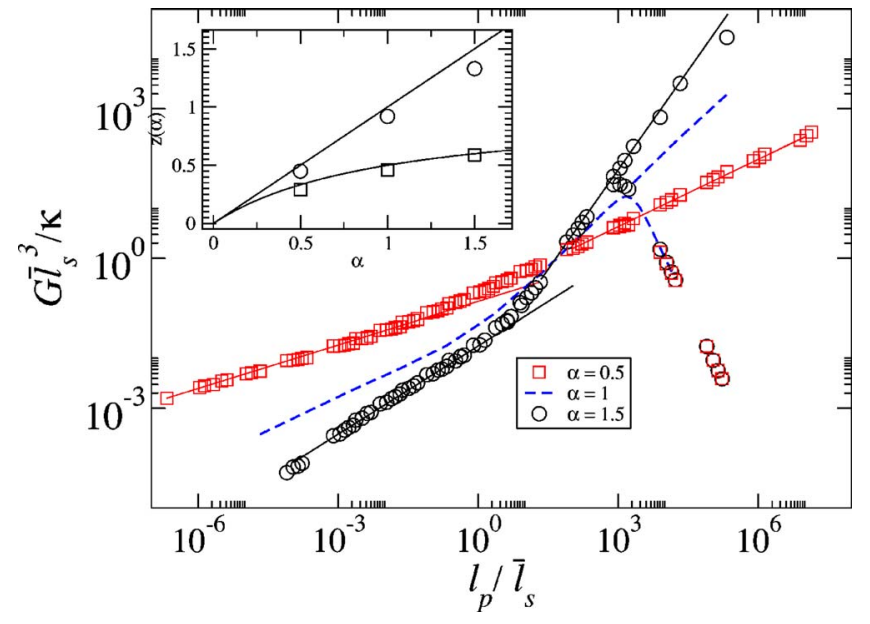

FIG. 11. (Color online) Scaling function $g$ as a function of $y$ $=l_{p} / \bar{l}_{s}$; the symbols correspond to values of $\alpha=0.5,1.5$. In addition the scaling function for $\alpha=1$ taken from Fig. 8 is shown (dashed line). Inset: exponents $z$ determined from the slopes of the branches $\mathrm{CL}_{\text {free }}$ (squares) and $U$ (circles), respectively. The solid lines correspond to the curves $z=\alpha$ and $z=\alpha /(1+\alpha)$ as derived in the main text.

ermost tails of the residual force distribution, will have sufficient energy to perturb the deformation field and relax to a state of lower strain.

In the following, we will assume that segments up to a critical length $l_{c}$ - to be determined self-consistently-fully relax from their affine reference state to give all their energy to the neighboring segment on the crossing filament. The total energy of the polymer can then be calculated from segments with $l_{s}>l_{c}$ only. There are two contributions: first, a stretching energy

$$
w_{s}\left(l_{s}\right) \simeq k_{\|} \delta_{\mathrm{aff}}^{2} \simeq \kappa \gamma^{2} \frac{l_{p}}{l_{s}^{2}}
$$

from the imposed affine strain field $\delta_{\text {aff }} \propto \gamma l_{s}$ and, second, a bending energy that is due to the relaxation of a neighboring segment on the crossing filament out of its affine reference state. This process requires that the segment of length $\hat{l}_{s}$ move the distance $\hat{\delta}_{\text {aff }}=\gamma \hat{l}_{s}$, which corresponds to its own affine deformation. The resulting bending energy

$$
w_{b}\left(l_{s}\right) \simeq k_{\perp} \hat{\delta}_{\mathrm{aff}}^{2} \simeq \kappa \gamma^{2} \frac{\hat{l}_{s}^{2}}{l_{s}^{3}}
$$

now depends on the length $l_{s}$ of the segment under consideration as well as on the length $\hat{l}_{s}$ of the neighboring (now relaxed) segment. As we have assumed above, the second contribution $w_{b}$ only arises if the length $\hat{l}_{s}$ is shorter than the critical length $l_{c}$. The total deformation energy along the polymer is then obtained by adding both contributions and integrating over all segments $l_{s}>l_{c}$ along the filament as well as averaging over neighbors with $\hat{l}_{s}<l_{c}$, 


$$
W_{\mathrm{pol}} \simeq\left(l_{f} \rho\right) \kappa \gamma^{2} \int_{l_{c}}^{\infty} d l_{s} P\left(l_{s}\right)\left(\frac{l_{p}}{l_{s}^{2}}+l_{s}^{-3} \int_{0}^{l_{c}} d \hat{l}_{s} P\left(\hat{l}_{s}\right) \hat{l}_{s}^{2}\right),
$$

where the prefactor $l_{f} \rho$ just counts the number of segments per polymer. For simplicity, we have not considered any dependence of the deformations on the orientation relative to the macroscopic strain field. In essence, this would only introduce some additional numerical prefactors that are irrelevant for the scaling picture developed here. The integrations are reparametrized by introducing the nondimensional variable $\lambda=\rho l_{s}$ such that we arrive at the expression for the average polymer energy:

$$
W_{\mathrm{pol}} \simeq \kappa \gamma^{2} l_{f} \rho^{2}\left(\rho l_{p} / \lambda_{c}+\lambda_{c}\right),
$$

where numerical constants have been dropped and $\lambda_{c}:=\rho l_{c}$ $\ll 1$ in the parameter range of interest. Minimizing with respect to $\lambda_{c}$ determines a new nonaffinity length

$$
l_{c}^{\min }=\lambda_{c}^{\min } \bar{l}_{s} \simeq \sqrt{l_{p} \bar{l}_{s}},
$$

which sets the maximal scale up to which the destruction of affine deformations lead to a lowering of the elastic energy. Inserting this length into Eq. (27) and multiplying by the number density of filaments $\rho / l_{f}$ one arrives at an expression for the modulus $G \simeq W_{\mathrm{pol}}^{\min } \rho / l_{f} \gamma^{2} \simeq \kappa \rho^{7 / 2} l_{p}^{1 / 2}$. Rewriting the result as

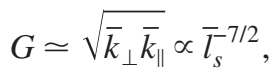

we immediately see that our theory reproduces the empirical result of Eq. (17) with an exponent $z=1 / 2$, which compares well with the measured value of $z=0.46$.

The nontrivial behavior of $G$ observed in Fig. 8 can thus be explained by a nonaffinity length scale $l_{c}^{\min } \simeq \sqrt{\bar{l}_{s} l_{p}}$ below which the affinity of the deformation field breaks down. Recapitulating the results from the cellular networks in Fig. 4, we observe that the same intermediate scaling behavior of $G \propto \bar{l}_{s}^{-7 / 2}$ is found in both architectures. We have thus established the microscopic origin of the scaling law. It derives from a continuous unloading of smaller segments driven by an interplay between segment length distribution and elastic properties of the single polymer. This mechanism is illustrated in Fig. 10, where a histogram for the fraction of energy stored in segments of various lengths is shown. For very small persistence length, a significant fraction of the energy is stored in the shortest segments. Affine deformations can be seen as a good approximation. Increasing the persistence length, the short segments one after the other lose their energies in favor of additional excitations in longer segments. This is fully consistent with the assumption of a growing nonaffinity scale $l_{c}^{\mathrm{min}}$ below which no energy is stored.

It is important to realize that our derivation of the exponent does not make use of the precise form of the segment length distribution $P\left(l_{s}\right)$. In fact, there is no need to perform the integrations explicitly and only the limiting behavior of $P\left(l_{s} \rightarrow 0\right)$ enters. Thus, the conclusions are valid for a general class of functions that may even be slowly vanishing at zero segment length.
We have also conducted simulations that assume a more general form for the stretching stiffness

$$
k_{\|}(\alpha)=6 \kappa \frac{l_{p}^{\alpha}}{l_{s}^{3+\alpha}},
$$

which reduces to the original definition for $\alpha=1$. Since the relative stiffness of the deformation modes is now $k_{\|} / k_{\perp}$ $\propto\left(l_{p} / l_{s}\right)^{\alpha}$, we can think of the phenomenological exponent $\alpha$ to tune the anisotropy of the individual segment. It allows us to extend our discussion to the broad class of systems for which $k_{\|}$is a monomial (with units energy per area) involving one additional material length $l_{p}$. Repeating the scaling theory for general values of $\alpha$ gives $z(\alpha)=\alpha /(1+\alpha)$ which is verified by the results of the simulations presented in Fig. 11. It provides further evidence for the validity of our scaling picture.

\section{Fixed cross-link angles}

If we want to apply the same reasoning to the network with the fixed cross-link angles, we face the problem that even a perfectly affine displacement of all the cross-links induces some amount of bending of the segments, in addition to the usual contribution from the stretching deformations. While an affine strain $\gamma$ would change all angles by an amount $\Delta \phi \propto \gamma$, due to the infinite rotational stiffness in the cross-links, this cannot actually occur. The segments therefore have to experience an extra bending contribution induced by cross-link rotations $(-\Delta \phi)$ that restore the angles to their original values. In the parameter regime $y \ll 1$, where bending is the stiffer mode, we therefore expect strong contributions to the energy from the bending mode already in the affine reference state. Allowing for the relaxation of the smallest segments from their stretched state to even stronger reduce the amount of stretching we might find an exponent as low as $z=0.07$, signaling nearly exclusive contributions from the bending mode, not too surprising. In fact, we will argue below that neglecting the stretching energies-i.e., assuming an exponent of $z=0$-represents a reasonable approximation to the elasticity in this regime.

\section{E. Universal regime}

By increasing $y$ from its small value we soften the bending mode and therefore reduce the influence of the fixed cross-link angles on the elastic energy. At the same time the nonaffinity scale $l_{c}^{\min } \propto y^{1 / 2}$ increases, indicating ever stronger deviations from the affine reference state. When, eventually, $l_{c}^{\min } \approx l_{p} \approx \bar{l}_{s}\left(\lambda_{c}^{\mathrm{min}} \approx y \approx 1\right)$, the affine strain field does not serve as a reference configuration any more, since it is strongly perturbed by a majority of segments with $l_{s}<l_{c}^{\min }$. At this stage, the two branches, present in the cross-linkdominated regime, merge and one enters a universal filament-dominated regime. There, the specific properties of the cross-links do not influence the macroscopic elasticity notably.

While the scaling argument presented for the hinged network ceases to be applicable, the remaining residual forces $\delta f$ continue to lead to a redistribution of stresses from shorter 
to longer segments, albeit at higher scales. As we have shown in [23], eventually about $90 \%$ of the energy is stored in the longest $30 \%$ of the segments only. The new feature as compared to the regime $\mathrm{CL}_{\text {free }}$ is that unloading of a segment from its stretched configuration will also lead to stretching of its immediate neighbor on the same filament [see Fig. 9(a)]. This way, the available energy for bending of the crossing filament, which was the primary contribution in Eq. (26), is reduced. In the limit $y \gg 1$ we can neglect these contributions and calculate the energy from the polymers' stretching stiffness only. The physical picture is that of a serial connection of infinitely many segments along the backbone of a "typical" polymer. The stiffness of this polymer, and therefore the modulus, may be obtained from the stretching spring constants of the individual segments $k_{\|}\left(l_{s}\right)$ as

$$
G^{-1}=\int d l_{s} P\left(l_{s}\right) k_{\|}\left(l_{s}\right)^{-1} \propto \bar{k}_{\|}^{-1},
$$

corresponding to the exponent $z=1$. For the more general response coefficient of Eq. (30) this argument predicts $z=\alpha$, a result which is closely confirmed by the results of the simulation as can be seen from the inset of Fig. 11. Note that the shear modulus in this asymptotic region takes the same form as postulated by the affine theory in Eq. (11). However, using Eq. (31) one can resolve the effects according to segment length to find that the contribution to the total energy from segments with length $l_{s}$ grows as $W\left(l_{s}\right) \propto l_{s}^{4}$. This strong increase is in accordance with the assumption of a large nonaffinity scale, below which no energy is stored, and in striking contrast to the affine theory that would yield $W_{\text {aff }}\left(l_{s}\right)$ $\propto k_{\|}\left(l_{s}\right) \delta_{\mathrm{aff}}^{2} \propto l_{s}^{-2}$.

\section{CONCLUSION}

We have studied the macroscopic elastic properties of networks of semiflexible polymers. We provide exhaustive numerical studies supplemented by scaling arguments that elucidate the subtle interplay between the architecture of the network and the elastic properties of its building blocks.

The main conclusion to be drawn is that, irrespective of the specific architecture chosen, thermally fluctuating stiff polymer networks are inherently more sensitive to polydispersity and randomness than their purely mechanical counterparts. This is due to their strongly length-dependent entropic stretching response $k_{\|}(l) \propto \kappa^{2} / l^{4}$ which has to be contrasted with the mechanical stretching stiffness $k_{s}(l)$ $\propto \kappa / l$.

Although simulations have only been conducted in twodimensional networks, the identified mechanism by which the structural randomness influences the elastic properties is expected to be of universal character and hold independent of dimensionality. As we have shown, the actual consequences of this susceptibility (e.g., scaling behavior of elastic moduli) may vary from system to system and certainly also with the dimension. A precise knowledge of the network architecture is therefore indispensable for the interpretation of experimental data. For this it will be most important to develop new techniques that allow the characterization of the microstructure and monitor its changes upon deformation. As exemplified by the discussion of the universal regime, Sec. IV E, where the (nonaffine) elastic modulus turns out to be similar to that in an affine theory, we have shown that macroscopic measurements alone do not suffice to extract the network mechanics also on the microscopic scale.

We have described how the polymer length $l_{f}$ can be used to drive the system from a simple cellular structure with filaments as short as the mesh size $l_{f} \approx \bar{l}_{s}$ to a fully scaleinvariant fibrous structure characterized by infinitely long filaments $l_{f} \rightarrow \infty$. Especially the latter limit allows for intricate scaling behavior that impressively demonstrates the qualitative difference between thermally fluctuating and purely mechanical elastic networks.

The elasticity of a simple cellular structure may be described by a serial connection of their elementary deformation modes bending and stretching, respectively. This leads to the modulus of Eq. (9),

$$
G^{-1}=a \bar{k}_{\perp}^{-1}+b \bar{k}_{\|}^{-1} .
$$

In this picture, deformations can be drawn from either mode and it will be the softer one that dominates the modulus. In fibrous networks with fixed cross-link angles we have shown that the modes rather act as if they were springs connected in parallel. The modulus can then be approximated by

$$
G=a \bar{k}_{\perp}+b \bar{k}_{\|}
$$

where the prefactors $a$ and $b$ depend weakly on the scaling variable $y \sim \bar{k}_{\|} / \bar{k}_{\perp} \sim l_{p} / \bar{l}_{s}$. The network elasticity is therefore always dominated by the stiffer mode, qualitatively similar to a triangulated network, where the specific geometry of the unit cell always imposes stretching deformations on the system, no matter how soft the bending mode actually is. The fibrous architecture apparently also suppresses the transition into regimes where the softer mode is dominant. This conclusion is consistent with recent simulations on the purely mechanical fiber model $[19,20]$, where a transition into a regime dominated by soft bending modes $(y \gg 1)$ could only be observed at finite values for the filament length $l_{f}$. Increasing the length to asymptotic values $l_{f} \rightarrow \infty$, as we have done here, such a "bending-soft" regime is strongly suppressed and eventually cannot occur anymore. Instead, the elasticity is governed by the much stiffer (mechanical) stretching mode. A detailed theoretical explanation of how this suppression is generated in mechanical fiber networks will appear elsewhere [44]; however, it is clear that the mechanism that leads to bending in cellular structures cannot work in fibrous networks. The fact that any segment is part of the larger structure of the polymer fiber leads to strong geometric correlations and imposes very strict conditions on possible segmental deformations.

Allowing the filaments to freely rotate at the cross-links, a situation which may be relevant for F-actin networks crosslinked, for example, with $\alpha$-actinin, we also find an asymptotic scaling regime where stretching and bending modes contribute equally to the elastic energy, Eq. (17), 


$$
G \propto \bar{k}_{\perp}^{1-z} \bar{k}_{\|}^{z} .
$$

By quantifying the degree of cooperation between neighboring elements in the network we were able to identify a nonaffinity length scale $l_{c}$ below which the state of affine deformations is rendered unstable. A scaling argument is supplied that allows the calculation of the effective macroscopic exponents starting from this microscopic picture.

It seems that the effects described above can only be accounted for by going beyond the conventional approach that considers typical polymer segments only. Instead, we propose to describe the elasticity in terms of a typical polymer filament and the spatial distribution of cross-links along its backbone. By controlling the architecture of the network, the scale of the polymer length $l_{f}$ therefore seems to implicitly influence the elastic properties of the system even in parameter regions where it does not enter the macroscopic elastic moduli explicitly.

\section{ACKNOWLEDGMENTS}

We gratefully acknowledge fruitful discussions with Mark Bathe, Oskar Hallatscheck, and Klaus Kroy.

\section{APPENDIX: STIFFNESS MATRIX}

This appendix derives an expression for the stiffness matrix of a polymer segment embedded in a two-dimensional network.
The differential equation governing the bending of a beam of length $l$ is given by $\kappa X^{(4)}=0$, where the transverse deflection $X$ is induced by the forces $F_{0}$ and $F_{l}$ as well as the torques $M_{0}$ and $M_{l}$ acting on both ends. The solution can then be written as

$$
X(s)=X_{0}+X_{0} s+\frac{s^{2}}{2 \kappa}\left(M_{0}-s F_{0} / 3\right),
$$

while equilibrium conditions require that

$$
F_{l}=-F_{0}, M_{l}=-\left(M_{0}-F_{0} l\right) .
$$

Stretching the beam to the position $Z$ is governed by the equation

$$
Z(s)=Z_{0}+s-\frac{s}{E A} T_{0},
$$

with the condition

$$
T_{l}=-T_{0},
$$

balancing the axial forces $T$.

The two variables $(X, Z)$ are the coordinates (in the frame of the fiber) of the vector $\mathbf{u}$ introduced in the main text. The rotation is given by $\theta=X^{\prime}$. The four equations (A1)-(A4) can now be inverted to yield the forces in terms of the displacements at the beam ends (cross-links):

$$
\left(\begin{array}{c}
F_{0} \\
T_{0} \\
M_{0} l \\
F_{l} \\
T_{l} \\
M_{l} l
\end{array}\right)=\frac{\kappa}{l^{3}}\left(\begin{array}{cccccc}
-12 & 0 & -6 & 12 & 0 & -6 \\
0 & \Lambda & 0 & 0 & -\Lambda & 0 \\
-6 & 0 & -4 & 6 & 0 & -2 \\
12 & 0 & 6 & -12 & 0 & 6 \\
0 & -\Lambda & 0 & 0 & \Lambda & 0 \\
-6 & 0 & -2 & 6 & 0 & -4
\end{array}\right)\left(\begin{array}{c}
X_{0} \\
Z_{0} \\
X_{0} l \\
X_{l} \\
Z_{l} \\
X_{l}^{\prime} l
\end{array}\right)
$$

where we have defined $\Lambda=l^{2} A / I=4(l / r)^{2}$. The second equality only holds for circular beam cross sections, where the moment of area $I=\pi r^{4} / 4$. The corresponding matrix is called the stiffness matrix.

If, in addition to Eq. (A3), we assume that the stretching response is governed by that of a thermally fluctuating stiff polymer, we have to take into account $k_{\|}$of Eq. (4). This is achieved by letting both stretching modes act in series and substitute $k_{s}^{-1} \rightarrow k_{s}^{-1}+k_{\|}^{-1}$. Equivalently, one can assign an effective polymer radius

$$
r_{p o l}^{2}=r^{2}+\frac{4 l^{3}}{\zeta l_{p}}
$$

which now depends on the segment length $l$ as well as on the persistence length $l_{p}$ of the polymer. 
[1] L. D. Landau and E. M. Lifshitz, Theory of Elasticity, 3rd ed. (Butterworth-Heinemann, Oxford, 1995), Vol. 7.

[2] B. Alberts et al., Molecular Biology of the Cell (Garland, New York, 1994).

[3] B. Hinner, M. Tempel, E. Sackmann, K. Kroy, and E. Frey, Phys. Rev. Lett. 81, 2614 (1998).

[4] J. Xu, A. Palmer, and D. Wirtz, Macromolecules 31, 6486 (1998).

[5] M. M. A. E. Claessens, R. Tharmann, K. Kroy, and A. R. Bausch, Nat. Phys. 2, 186 (2006).

[6] T. Odjik, Macromolecules 16, 1340 (1983).

[7] W. Helfrich and W. Harbich, Chem. Scr. 25, 32 (1985).

[8] H. Isambert and A. C. Maggs, Macromolecules 29, 1036 (1996).

[9] A. P. Philipse, Langmuir 12, 1127 (1996).

[10] D. Rodney, M. Fivel, and R. Dendievel, Phys. Rev. Lett. 95, 108004 (2005).

[11] S. R. Williams and A. P. Philipse, Phys. Rev. E 67, 051301 (2003).

[12] J. H. Shin et al., Proc. Natl. Acad. Sci. U.S.A. 101, 9636 (2004).

[13] M. Tempel, G. Isenberg, and E. Sackmann, Phys. Rev. E 54, 1802 (1996).

[14] O. Pelletier, E. Pokidysheva, L. S. Hirst, N. Bouxsein, Y. Li, and C. R. Safinya, Phys. Rev. Lett. 91, 148102 (2003).

[15] R. Tharmann, M. M. A. E. Claessens, and A. R. Bausch, Biophys. J. 90, 2622 (2006).

[16] L. Limozin and E. Sackmann, Phys. Rev. Lett. 89, 168103 (2002).

[17] I. Borukhov, R. F. Bruinsma, W. M. Gelbart, and A. J. Liu, Proc. Natl. Acad. Sci. U.S.A. 102, 3673 (2005).

[18] A. G. Zilman and S. A. Safran, Europhys. Lett. 63, 139 (2003).

[19] J. Wilhelm and E. Frey, Phys. Rev. Lett. 91, 108103 (2003).

[20] D. A. Head, A. J. Levine, and F. C. MacKintosh, Phys. Rev. Lett. 91, 108102 (2003).

[21] D. A. Head, A. J. Levine, and F. C. MacKintosh, Phys. Rev. E 68, 061907 (2003).

[22] P. R. Onck, T. Koeman, T. van Dillen, and E. van der Giessen, Phys. Rev. Lett. 95, 178102 (2005).

[23] C. Heussinger and E. Frey, Phys. Rev. Lett. 96, 017802
(2006).

[24] E. Frey, K. Kroy, J. Wilhelm, and E. Sackmann, in Dynamical Networks in Physics and Biology, edited by G. Forgacs and D. Beysens (Springer, Berlin, 1998), Chap. 9.

[25] J. A. Åström, J. P. Mäkinen, M. J. Alava, and J. Timonen, Phys. Rev. E 61, 5550 (2000).

[26] M. Sahimi, Heterogeneous Materials (Springer, New York, 2003).

[27] K. Kroy and E. Frey, Phys. Rev. Lett. 77, 306 (1996).

[28] F. C. MacKintosh, J. Käs, and P. A. Janmey, Phys. Rev. Lett. 75, 4425 (1995).

[29] D. Weaire and S. Hutzler, The Physics of Foams (Oxford University Press, Oxford, 2001).

[30] L. J. Gibson and M. F. Ashby, Cellular Solids: Structure and Properties (Cambridge University Press, Cambridge, England, 1999).

[31] A. M. Kraynik and W. E. Warren, in Low Density Cellular Plastics, edited by H. Hilyard and C. Cunningham (Kluwer Academic, Amsterdam, 1994), Chap. 7.

[32] H. X. Zhu, J. F. Knott, and N. J. Mills, J. Mech. Phys. Solids 45, 319 (1997).

[33] M. J. Silva, W. C. Hayes, and L. J. Gibson, Int. J. Mech. Sci. 37, 1161 (1995).

[34] H. X. Zhu, J. R. Hobdell, and A. H. Windle, Adv. Mater. (Weinheim, Ger.) 48, 4893 (2000).

[35] A. Fazekas, R. Dendievel, L. Salvo, and Y. Bréchet, Int. J. Mech. Sci. 44, 2047 (2002).

[36] D. Boal, Mechanics of the Cell (Cambridge University Press, Cambridge, England, 2002).

[37] O. Kallmes and H. Corte, Tappi J. 43, 737 (1960).

[38] J. A. Åström, S. Saarinen, K. Niskanen, and J. Kurkijärvi, J. Appl. Phys. 75, 2383 (1994).

[39] M. Latva-Kokko and J. Timonen, Phys. Rev. E 64, 066117 (2001).

[40] S. Heyden, Ph.D. thesis, Lund University, 1996.

[41] R. L. Satcher and C. F. Dewey, Biophys. J. 71, 109 (1996).

[42] C. Storm et al., Nature (London) 435, 191 (2005).

[43] M. L. Gardel et al., Science 304, 1301 (2004).

[44] C. Heussinger and E. Frey, Phys. Rev. Lett. 97, 105501 (2006). 